\title{
STM observations of a one-dimensional electronic edge state at steps on $\mathrm{Cu}(111)$
}

\author{
Ludwig Bartels \\ University of California at Riverside, Riverside, California, 92521 \\ S. W. Hla \\ Department of Physics and Astronomy, Ohio University, Athens, Ohio 45701 \\ A. Kühnle, ${ }^{*}$ G. Meyer, ${ }^{\dagger}$ and K.-H. Rieder \\ Fachbereich Physik, Freie Universität Berlin, Arnimallee 14, D-14195 Berlin, Germany \\ J. R. Manson \\ Department of Physics and Astronomy, Clemson University, Clemson, South Carolina 29634 \\ (Received 9 September 2002; revised manuscript 2 January 2003; published 23 May 2003)
}

\begin{abstract}
Scanning tunneling microscopy measurements across isolated straight step edges on a $\mathrm{Cu}(111)$ surface were carried out for biases between $100 \mathrm{mV}$ and $5 \mathrm{~V}$. In addition to the well known surface state oscillations, and at lower sample bias than the onset of the two-dimensional surface image state, a sharply defined linear protrusion, was observed at the top of the step faces. This linear feature is interpreted as a one-dimensional image state at the step, with its energy modified by a dipolar potential whose appearance is attributed to Smoluchowski smoothing of the electron density at the step edge.
\end{abstract}

DOI: 10.1103/PhysRevB.67.205416

PACS number(s): 73.20.-r, 73.61.-r

The electronic structures of clean surfaces of noble metals are among the best studied aspects of surface science. This makes them especially well suited for searches of new phenomena and new properties continue to be found: Two examples are the striking images of standing wave oscillations found by the groups of Avouris ${ }^{1}$ and Eigler ${ }^{2}$ and the remarkable experiments of Höfer $\mathrm{et} \mathrm{al.}{ }^{3}$ showing that image state (IS) electrons can penetrate hundreds of $\AA$ into the vacuum. Here we report on a related feature: a one-dimensional (1D) density of states, which we attribute to the occurrence of a 1D IS at the step edge (SE).

Recently, the resolution of surface science experiments has increased dramatically. The dispersion of surface states (SS) has been measured with unprecedented precision using photoemission spectroscopy, ${ }^{4}$ and several scanning tunneling microscopy (STM) studies have produced results of remarkable resolution. ${ }^{5}$ The knowledge about IS has vastly increased thanks to nonlinear laser spectroscopy. ${ }^{6,7}$ These results have in common that they allow comparison between essentially quantum mechanical, multiparticle effects, and their (semi)classical analogies, e.g., using concepts derived from macroscopic physics for the description of standing waves in the charge density patterns in confined SS.

For our studies we used a self-built STM operated at $15 \mathrm{~K}$ with electrochemically etched tungsten wire as tips, and a $\mathrm{Cu}(111)$ crystal prepared by several cycles of sputtering and annealing. We prepared the tip in situ by gently dipping it into the substrate and consequently it is assumed to be covered with $\mathrm{Cu}^{8}$ Figure 1(a) shows an STM image of two terraces intersected by a long, straight trench of $\simeq 40 \AA$ in width. On the higher-lying terraces the familiar SS oscillations $^{1,2}$ can be seen. Figure 1(b) illustrates the measured tip heights ( $z$ axis) as a function of the bias voltage ( $y$ axis). All scans lie along the same path normal to the trench in Fig. 1(a) which is shown as the $x$ axis. During the mea- surement the tip was repeatedly scanned along the same path across the step in a constant current mode. Positive sample bias was used with a different bias applied at each scan and the corresponding tip height was recorded. Scans corresponding to biases between $100 \mathrm{meV}$ and $5 \mathrm{~V}$ are plotted as a map in Fig. 1(b). Figure 1(c) shows cross sectional cuts from Fig. 1(b) of tip height versus distance perpendicular to the steps at six different bias values. Very striking in Fig. 1(b) is the steep rise of the apparent tip height between 4 and $5 \mathrm{~V}$. An increase in tip-height in the constant current mode would indicate that, in this voltage range, if the tip were held at constant height the current would increase. This is attributed to electron tunneling into the surface IS of $\mathrm{Cu}(111){ }^{9,10}$ Even more striking is the ridge parallel to the SE indicated with white arrows. Features associated with steps and attributed to edge electronic states have been observed previously, ${ }^{11}$ but this protrusion appears to be a new observation. It cannot be attributed to an overcompensation of the SE by the feedback loop as it appears symmetrically on both sides of the trench.

The most obvious explanation of the origin of this ridge might, at first glance, appear to be reflections of the SS at the SE. Indeed, it has been pointed out ${ }^{1,2}$ that if a step edge is modeled by a repulsive hard barrier, in spite of the fact that the wave function must vanish at the position of the barrier, just in front of the barrier all partial waves of the electrons in the SS corresponding to different $k_{\|}$values add up and contribute constructively to the probability density. Thus one expects a maximum in the SS density just in front of the step edge, and this maximum should sharpen and approach nearer to the step edge with increasing bias. As is apparent from Figs. 1(a) and 1(b), the protrusion observed here is much larger than any of the oscillations due to the SS indicating an alternative origin at higher bias. We attribute this feature to a localized linear state of an electron trapped in its own polar- 
a)
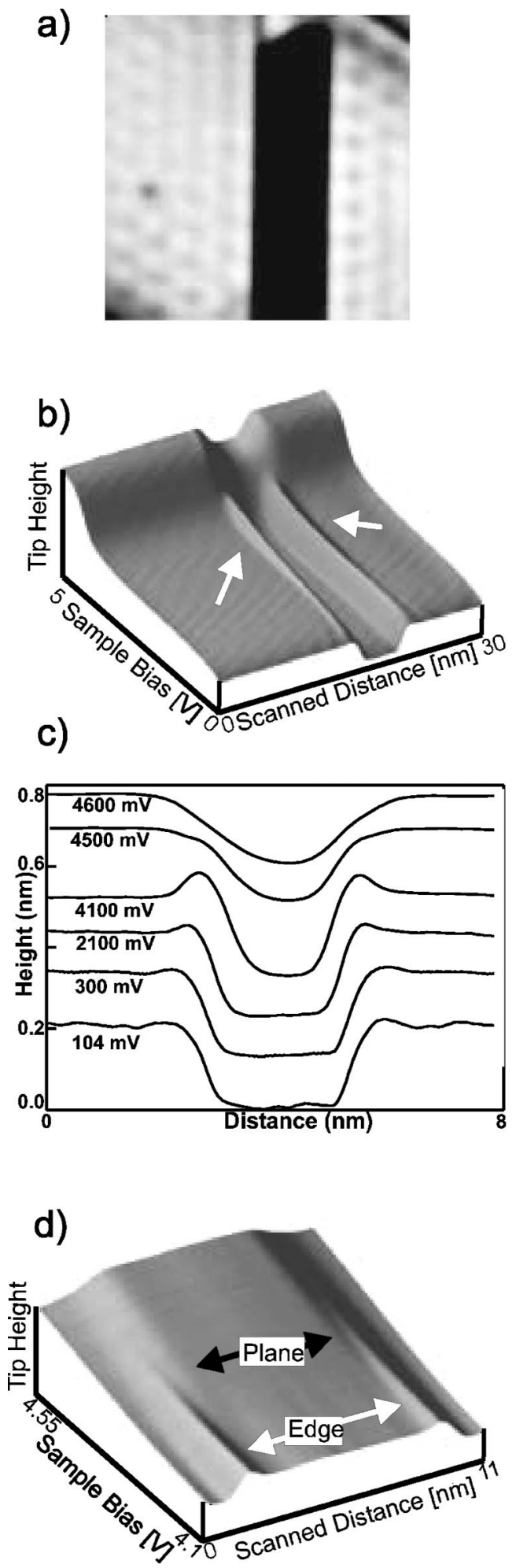

FIG. 1. (a) An STM image of two terraces on $\mathrm{Cu}(111)$ intersected by a long, straight trench. ( $180 \AA \times 180 \AA$, with a tip bias of $35 \mathrm{mV}$.) (b) Scans taken along a fixed straight line normal to the trench always at the same spatial position but with different biases. (c) Cross-sectional cuts at six different bias voltages of (b) showing tip height versus distance perpendicular to the steps. (d) Similar to (b) except that it shows scans over a reduced bias range and across a ridge found at a different location on the same $\mathrm{Cu}(111)$ sample. ization potential near the step edge and also under the influence of the linear dipolar potential present at a step. This is the $1 \mathrm{D}$ analog of the 2D surface IS. The lateral extent of such a feature would be expected to be governed by a decay length roughly similar to the 4 atomic units of a terrace IS, and Fig. 1(c) corroborates this. For example, at a bias of 4.1 $\mathrm{eV}$ the lateral extension of the 1D IS onto the terrace has a half-width at half-maximum of approximately 4.2 atomic units.

While the discrepancy between the observed broadening of the 1D feature and the expected sharpening of an SS reflection at the step edge might still be attributed to an effect of declining spatial resolution of the STM tip with increasing bias, in order to stem from the SS the protrusion would also have to rise proportionately to the height of the plane which is not observed, as shown in Fig. 2. Figure 2(a) shows the tip heights over both the terrace plane and at the SE protrusion as functions of increasing bias. Subtracting the height of the terrace from the height of the protrusion, the dashed line in Fig. 2(b) is obtained (0.8 nA data). Note that the apparent decrease of this peak height above $3.7 \mathrm{~V}$ is caused by the rapid increase of the terrace plane height as a consequence of the sudden onset of the IS, and does not signify an absolute decrease of the protrusion height.

In order to eliminate this effect from the data, the tip height due to the SS was computed using a SS band edge $\left(E_{0}\right)$ of $-0.43 \mathrm{eV}$ relative to the Fermi energy $E_{f}=$ $-4.93 \mathrm{eV}$ (Ref. 4) and an effective mass of 0.45 (Ref. 4) in the standard theory of the tunneling junction. ${ }^{12,13}$ The only free parameter of the calculation is the scaling parameter of the local density of states of the tip relative to that of the substrate. In Fig. 2(c) the calculated tip height (dashed curve) is compared to the measured value (solid curve). Given the simplicity of the model a remarkably good correlation of the two curves is obtained for biases below $3.5 \mathrm{~V}$, above which the terrace IS begins to appear. Readjusting the relative density of states to make the calculated curve match the height of the protrusion at low voltages we are able to predict how the protrusion height should increase if it were due only to the SS. The solid curve of Fig. 2(b) shows the difference between the calculated and the measured protrusion height. Below approximately $1.5 \mathrm{~V}$ the difference is virtually zero, while above that value a marked increase of the tip height in the vicinity of the SE is observed, which is not caused by the $\mathrm{SS}$ and hence must be attributed to a different phenomenon.

On many metal surfaces a series of IS can be found, which arise due to the charge redistribution in the substrate in response to an electron near the surface. To a good approximation such a series of IS follows a Rydberg series at $1 / 16$ of the energetic level spacing found for the hydrogen atom. On $\mathrm{Cu}(111)$ the first or lowest energy IS (henceforth simply referred to as the IS) is found to lie at $0.83 \mathrm{eV}$ below $E_{\text {vac }}$ [a little higher than the expected value of $13.6 \mathrm{eV} / 16$ $=0.85 \mathrm{eV}$ (Ref. 14)]. We simulate the position of the IS on the terrace plane by solving the 1D Schrödinger equation with the inclusion of the tip induced bias potential

$$
\left(-\frac{\hbar^{2}}{2 m_{e}} \frac{d^{2}}{d z^{2}}-\frac{1}{16 \pi \varepsilon_{0}} \frac{e^{2}}{z}+U(z)-E\right) \Psi_{\mathrm{IS}}=0,
$$




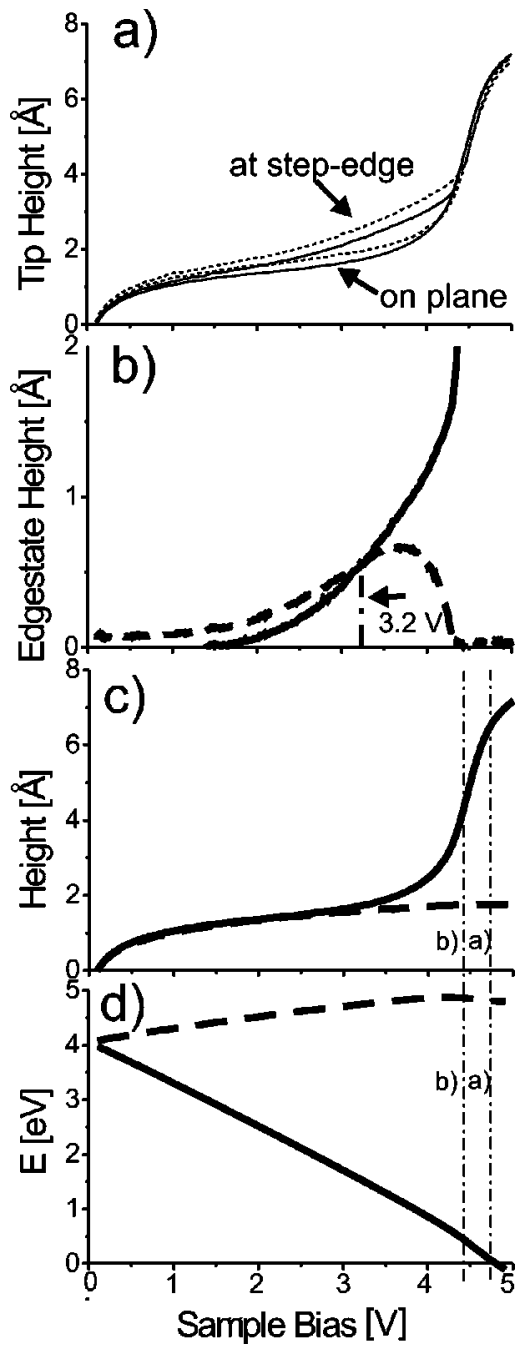

FIG. 2. (a) shows the variation of the tip height both at the SE and on the terrace as a function of changing sample bias at currents of $0.8 \mathrm{nA}$ (solid lines) and $3.0 \mathrm{nA}$ (dashed lines). The dashed line in (b) shows the difference between the two solid lines of (a), i.e., the measured dependence of the protrusion height relative to terrace plane as a function of the sample bias. The solid line indicates the height of the protrusion with reference to a simulated tip height on the terrace, which is shown as the dashed curve in (c) together with the measured tip height over the terrace plane at $0.8 \mathrm{nA}$ (solid line). (d) The energetic position of the IS with respect to the Fermi energy $E_{F}$ as a function of the applied bias and the correspondingly measured tip height (dashed line). The energetic difference between the IS center and the Fermi level of the tip (determined by the applied bias) is indicated with the solid line.

where $m_{e}$ is the electron mass, $e$ is the electron charge, $\hbar$ is Planck's constant divided by $2 \pi, \epsilon_{0}$ is the permittivity of free space, and $U(z)=U_{I}(z)=(\operatorname{bias} \times z) /$ [tip height (bias)], is the linear potential due to the tip induced bias. The result of such a calculation is the dashed curve of Fig. 2(d). This is a lowest order theoretical result and is clearly independent of any material specific corrections. ${ }^{14}$ The solid line in Fig. 2(d) represents the energetic difference between the electrons of maximum energy ejected from the tip at a given bias and $E_{0}$ of the IS. At a bias

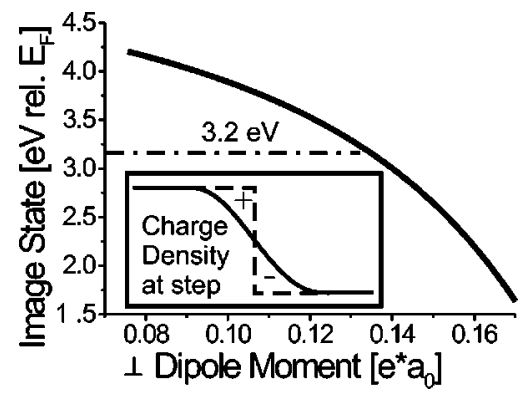

FIG. 3. The inset illustrates the smearing out of the charge density at a SE according to the Smoluchowski smoothing effect, leading to a local dipole with a component normal to the surface. The solid curve shows the calculated dependence of the energetic position of the 1D IS on the normal component of that dipole moment. A sample bias of $3.2 \mathrm{~V}$ and the corresponding measured tip height $(I=0.8 \mathrm{nA})$ were assumed.

of $4.8 \mathrm{~V}$ [marked (a) in Figs. 2(c) and 2(d)] $E_{0}$ of the IS starts to lie below the Fermi energy of the tip and the tipheight begins to saturate. At the bias point of $4.4 \mathrm{~V}$, marked (b) in Figs. 2(c) and 2(d), half of the steep increase of the tip height is reached when the difference between the applied bias and the IS is less than $0.5 \mathrm{~V}$. Both results strongly confirm that the observed increase of the tip height above $4.5 \mathrm{~V}$ at all lateral positions on the terrace corresponds to the IS. ISs have been observed using STM as early as $1985 .{ }^{9,10}$ Furthermore, in a number of studies by the group of Himpsel the difference between the energetic position of the IS on strands of metals adsorbed at SE's relative to the substrate itself was used to resolve their extent. ${ }^{15}$ Although the strands which they studied naturally occurred at SE's, properties of pure surfaces were not reported.

There is a considerable body of literature dealing with the confinement of SS and IS on regularly stepped surfaces. ${ }^{16,17}$ Generally, the steps cause a slight upshift of their energies accompanied by a change of their dispersion and possible correlation effects between neighboring terraces. Their results are, however, in general not applicable to isolated SE's.

Performing STM at positive but low sample voltage, the majority of the tunneling current is injected into the SS rather than the $s p$ band, as the latter does not offer any suitable final states with $k$ value perpendicular to the surface ( $L$ gap of copper). The validity of this assumption is supported by the good agreement of the simulations of Fig. 2(c) which do not take the $s p$ band into account at all. Avouris et al. ${ }^{16}$ suggested as an explanation for their experiments at low sample voltage that the local break of symmetry at an SE may modify this. While the authors cannot rule out effects of the $s p$ band on the data presented, they found no way to correlate the specific effect observed with the broken symmetry at a step edge. The following, however, describes how the data can be understood in terms of a 1D image state along the step edge.

As early as 1941 Smoluchowski $^{18}$ explained the lowering of the work function of stepped surfaces by the smearing out of charge density at steps (inset in Fig. 3), which results in a dipole with a component normal to the surface. This reduction in work function has recently been confirmed by STM 
experiments. ${ }^{19}$ Thus near an SE, electrons in front of the surface experience, in addition to the attraction due to the classical image charge, another force due to this dipole. This can lead to a quite significant local change in the energetic position of the IS. Again, Eq. (1) is used to calculate the energy of the IS but now the normal component of the dipole moment is included through the term $U(z)=U_{I}(z)+U_{I I}(z)$ where $U_{I I}(z)=-(p e) /\left(4 \pi \varepsilon_{0} z^{2}\right)$. The result of such a calculation is the shift of the image state as shown in Fig. 3 where the new energy of the IS state relative to $E_{F}$ is plotted as a function of dipole moment strength $p$. Assuming an applied sample bias of $3.2 \mathrm{~V}$ [where the height of the SE protrusion is already quite significant as seen in Fig. 2(b)] an effective dipole moment of $0.13 e a_{0}$ (where $a_{0}$ is the Bohr radius) is necessary to place $E_{0}$ of the SE IS at the corresponding energetic position. Such a dipole moment compares well with what is found at chemisorbed molecules on surface $^{20}$ and is far more conservative than the one assumed by Smoluchowski. ${ }^{18}$ It can also be compared with the minimum dipole moment $p=0.639 e a_{0}$ required to bind an electron ${ }^{21}$ without the background image charge. ${ }^{22}$

Finally, a comparison of the assumed dipole moment of $0.13 e a_{0}$ with the measured shift of the work function between the (111) surface and regularly stepped surfaces [e.g., $\mathrm{Cu}(211)]$ is in order. Two separate work function calculations were carried out, one assuming a dipole moment of $0.13 e a_{0}$ residing in each unit cell of a $\mathrm{Cu}(211)$ surface, and the other assuming a linear dipole wire. The results of both calculations were very similar, producing shifts of $0.75-0.8 \mathrm{eV}$ as compared to the measured value of $0.4 \mathrm{eV}$ for $\mathrm{Cu}(211){ }^{23}$ Given that all effects of the strong relaxation and reconstruction of the $\mathrm{Cu}(211)$ surface $^{24}$ and the spreading out of charge into the vacuum at the uncorrugated surface are not taken into account (both of which contribute corrections to the work function of the same order of magnitude but of opposite $\operatorname{sign}^{18}$ ), the match between calculation and experiment is significant.

Note that according to this model the protrusion originates from a strictly 1D, quantum-wire-like IS. No additional, conventional IS would be expected at this point. This is confirmed by Fig. 1(d) which shows in detail the rise of the IS on the plane between two SE protrusions found on a long, straight ridge on $\mathrm{Cu}(111)$. Clearly it can be seen that the flat-surface IS on the plane (black arrows) arises separately from the protrusion at the SE (white arrows) and only overshadows it when the tip sharpness becomes insufficient to resolve both states. ${ }^{25}$
Having put forward the idea of a 1D IS, it is now necessary to explain why it has not been observed previously, for instance by two-photon photoemission which usually has a much better energy resolution in this range. The answer may lie not only in the small coverage of the sample surface with SEs and their associated dipoles which are interspersed by (wide) terraces, but also in the fact that a quantum-wire-like state has no $k$ vector orthogonal to the wire in the surface plane. This allows electrons in it to propagate freely into the $s p$ band off the Brillouin zone center, making them invisible to angle-resolved photoemission and leading to an ultrashort lifetime of the 1D state itself which diminishes the yield of two-photon based excitation processes, as these rely on the stability of the electron population between the first and second excitation. However, STM yield is rather increased by a short lifetime of a state, as a multitude of fast decay channels provides an increase in the tunneling probability. A related issue is the recently reported indirect evidence for the presence of spectroscopical dark states responsible for desorption of adsorbates. ${ }^{26}$ In addition, assuming a reduction of lifetime from a typical $18 \mathrm{fs},{ }^{27,28}$ to a significantly lower value, the lifetime broadening becomes large ${ }^{29}$ making such a state harder to resolve from the background. Such a strong lifetime broadening would also explain the onset of the protrusion at comparatively low bias as observed in Fig. 2(b), since broadening of the energy level allows tunneling into the state at smaller biases. Similar broadening causes the observation of the IS in Fig. 1 at biases smaller than its most probable energy.

In conclusion, using STM we have found an elongated feature parallel to SE's on $\mathrm{Cu}(111)$. It was observed over a large range of positive sample bias from about 1.5 to $4 \mathrm{~V}$, above which it submerges in the regular IS. This feature can be explained as a localized 1D (quantum-wire-like) IS originating from the dipole moment of SE's arising as a consequence of edge polarization and Smoluchowski smoothing of the electron density. Thus, a new state of $1 \mathrm{D}$ character is found on a surface as simple as $\mathrm{Cu}(111)$.

J.R.M. and L.B. wish to express their thanks for the financial support of DFG, FES, and Leopoldina. This project was partly funded by the DFG under Grant No. RI 472/3-1 (K.-H. R.); the NSF under grant No. DMR 0089503 (J. R. M.); the NSF under Grant No. CHE 0210833/DMR 0116339, the DOE under Grant No. BES22436, and the ACS PF type G (L. B.). Useful discussions with F. Forstmann, B. Hamprecht, M. Wolf, E. Knoesel, and A. Hotzel are gratefully acknowledged.
*Present address: Institute of Physics and Astronomy, University of Aarhus, DK-8000 Aarhus, Denmark.

†Present address: IBM Zurich Research Laboratories, 8803 Rüschlikon-ZH, Switzerland.

${ }^{1}$ Y. Hasegawa and Ph. Avouris, Phys. Rev. Lett. 71, 1071 (1993).

${ }^{2}$ M. F. Crommie, C. P. Lutz, and D. M. Eigler, Nature (London) 363, 524 (1993).

${ }^{3}$ U. Höfer, I. L. Shumay, Ch. Reuss, U. Thomann, W. Wallauer, and Th. Fauster, Science (Washington, DC, U.S.) 277, 1480 (1997); A. Eiguren et al., Phys. Rev. Lett. 88, 066805 (2002).
${ }^{4}$ R. Paniago, R. Matzdorf, G. Meister, A. Goldmann, Surf. Sci. 331-333, 1233 (1995).

${ }^{5}$ J. T. Li et al., Phys. Rev. Lett. 81, 4464 (1998); L. Burgi et al., ibid. 81, 5370 (1998); M. Bode, M. Getzlaf, and R. Wiesendanger, ibid. 81, 4256 (1998); C. Wittneven et al., ibid. 81, 5616 (1998); V. Madhavan et al., Science (Washington, DC, U.S.) 280, 567 (1998).

${ }^{6}$ E. Knoesel, A. Hotzel, and M. Wolf, Phys. Rev. B 57, 12812 (1998).

${ }^{7}$ S. Ogawa et al., Phys. Rev. Lett. 78, 1339 (1997). 
${ }^{8}$ L. Bartels, G. Meyer, and K.-H. Rieder, Phys. Rev. Lett. 79, 697 (1997).

${ }^{9}$ G. Binnig et al., Phys. Rev. Lett. 55, 991 (1985).

${ }^{10}$ R. S. Becker, J. A. Golovchenko, and B. S. Swartzentruber, Phys. Rev. Lett. 55, 987 (1985).

${ }^{11}$ P. Avouris and I.-W. Lyo, Science (Washington, DC, U.S.) 264, 942 (1994).

${ }^{12}$ J. Tersoff and D. R. Hamann, Phys. Rev. Lett. 50, 1998 (1983).

${ }^{13}$ N. D. Lang, Phys. Rev. Lett. 55, 230 (1985).

${ }^{14}$ W. Steinmann and Th. Fauster, in Laser Spectroscopy and Photochemistry on Metal Surfaces, edited by H.-L. Dai and W. Ho (World Scientific, Singapore, 1994).

${ }^{15}$ T. Jung, Y. W. Mo, and F. J. Himpsel, Phys. Rev. Lett. 74, 1641 (1995).

${ }^{16}$ J. E. Ortega et al., Phys. Rev. B 49, 13859 (1994): O. Sanchez et al., ibid. 52, 7894 (1995); X. Y. Wang, X. J. Shen, and R. M. Osgood, Jr., ibid. 56, 7665 (1997); J. M. Garcia et al., Appl. Phys. A: Mater. Sci. Process. 61, 609 (1995); S. Crampin, M. H. Boom, and J. E. Inglesfield, Phys. Rev. Lett. 73, 1051 (1994).

${ }^{17} \mathrm{Ph}$. Avouris, I.-W. Lyo, and P. Molinas-Mata, Chem. Phys. Lett. 240, 423 (1995).

${ }^{18}$ R. Smoluchowski, Phys. Rev. 60, 661 (1941).

${ }^{19}$ J. F. Jia, K. Inoue, Y. Hasegawa, W. S. Yang, and T. Sakurai,
Phys. Rev. B 58, 1193 (1998).

${ }^{20}$ For example, $0.75 e a_{0}$ for $\mathrm{NH}_{3} / \mathrm{Ru}(001)$; C. Benndorf and T. E. Madey, Surf. Sci. 135, 164 (1983), and $0.098 e a_{0}$ for CO/ Cu(100); R. Ryberg, Phys. Rev. B 32, 2671 (1985).

${ }^{21}$ E. Fermi and E. Teller, Phys. Rev. 72, 406 (1947).

${ }^{22}$ Calculations were also carried out for the dipolar potential $U_{I I}$ replaced by a second imagelike potential $U_{I I}=V_{0} a_{0} / z$, as, for example, expected for a charge interacting with a linear dipole wire, and the results are quite similar to those of Fig. 3 when plotted as a function of the strength $V_{0}$.

${ }^{23}$ P. O. Gartland et al., Phys. Norv. 7, 39 (1973) measured $4.53 \mathrm{eV}$ for the work function of $\mathrm{Cu}(211)$ compared to $4.93 \mathrm{eV}$ for $\mathrm{Cu}(111)$ according to Ref. 4.

${ }^{24}$ C. Y. Wei et al., Phys. Rev. B 57, 10062 (1998).

${ }^{25}$ Compare the maximum slope on the outward edge of the protrusion to find its resolution power.

${ }^{26}$ L. Cai, X. D. Xiao, and M. M. T. Loy, Surf. Sci. 492, L688 (2001).

${ }^{27}$ M. Wolf, Surf. Sci. 377-379, 343 (1997).

${ }^{28}$ E. V. Chulkov, I. Sarria, V. M. Silkin, J. M. Pitarke, and P. M. Echenique, Phys. Rev. Lett. 80, 4947 (1998).

${ }^{29} \mathrm{At} \simeq 1$ fs the minimum FWHM is almost $1 \mathrm{eV}$. 